Original Research Paper

\title{
Galactomannan Restore Letrozole Induced Polycystic Ovarian Syndrome (PCOS) in Rat Model
}

\author{
${ }^{1}$ Manoj K. Aswar, ${ }^{1}$ Utkarsha V. Deshpande and ${ }^{2}$ Urmila Aswar \\ ${ }^{1}$ Department of Pharmacology, Sinhgad Institute of Pharmacy, Narhe, Pune \\ ${ }^{2}$ Department of Pharmacology, Bharati Vidyapeeth's Poona College of Pharmacy, Erandwane, Pune
}

\author{
Article history \\ Received: 11-09-2021 \\ Revised: 19-10-2021 \\ Accepted: 24-10-2021 \\ Corresponding Author: \\ Manoj K. Aswar \\ Department of Pharmacology, \\ Sinhgad Institute of Pharmacy, \\ Narhe, Pune \\ Email: aswar.manoj@gmail.com
}

\begin{abstract}
Current research aims towards evaluation of the effect of Galactomannan (GM) on letrozole-induced PCOS in adult female Wistar rats. Seven randomized groups of six female rats were made. The rats in all groups were administered with letrozole ( $1 \mathrm{mg} / \mathrm{kg}$ p.o.) for 21 days to induce PCOS. Rats in respective groups were treated with vehicle $(10 \mathrm{ml} / \mathrm{kg}$, p.o.), clomiphene citrate (1 mg/kg, p.o.), galactomannan (10, 20, 40 $\mathrm{mg} / \mathrm{kg}$, p.o. $)$ and clomiphene citrate + galactomannan $(1+20 \mathrm{mg} / \mathrm{kg}$, p.o. $)$ from day 22 to 36 . On the last day of treatment i.e., $37^{\text {th }}$ day of the study, blood was withdrawn, serum was separated and was used to assess hormonal and lipid profile. The rats were sacrificed in the late diestrus stage, ovaries were isolated and used for histopathological studies. Cysts in the ovaries of rats in control group was clearly seen after giving letrozole. Treatment either with Galactomannan or clomiphene citrate, significantly decreased blood sugar level, lipid profile, LH and testosterone whereas increased concentration of FSH and AMH was observed at the end of study. Reduced number of ovarian cysts as well as decreased body weight and ovary weight was documented for the possible protective role of galactomannan in PCOS. On the basis of results, galactomannan exerts protective effect in PCOS and could be given as adjuvant treatment with the available pharmacotherapy for the management of PCOS in women.
\end{abstract}

Keywords: Clomiphene Citrate, Galactomannan, Insulin Sensitivity, Letrozole, PCOS

\section{Introduction}

PCOS is a common endocrine disorder among female reproductive population, with a prevalence of about $2-7 \%$. Its clinical manifestations include irregular menses and hyperandrogenimia associated with metabolic dysfunction in which hyperinsulinemia and peripheral insulin resistance are seen (Nair et al., 2012). During the reproductive years, PCOS is associated with significant reproductive morbidity including infertility, abnormal uterine bleeding, miscarriage and other complications of pregnancy (Wild, 2002). It has been well documented that inappropriate gonadotropin secretion, especially high Luteinizing Hormone (LH) secretion, is associated with PCOS with the drastic change in LH: FSH ratio in PCOS patients (Norman et al., 2007). Patients with PCOS have other metabolic complications such as dyslipidemia (abnormal serum lipid levels), hypertension, insulin resistance and endothelial dysfunction. Almost 70\% PCOS women have dyslipidemia, out of which, $50 \%$ women are obese. Furthermore, dyslipidemia induced atherosclerosis is the primary cause for development of cardiovascular diseases (Expert Panel on Detection, 2001; Wild, 2002). Insulin resistance and dyslipidemia are concurrently observed in most PCOS women which lead to hyperglycemia and the associated complications (Maqbool et al., 2019). Hyperinsulinemia, resulting from insulin resistance plays a pathogenic role in PCOS by stimulating ovarian testosterone production, decreasing serum sex hormone binding globulin concentrations and impending ovulation (Legro et al., 2005). Hence combination therapies of insulin sensitizing drugs such as metformin and thiazolidinediones with lipid lowering drugs such as statins, nicotinic acid and fibric acid derivatives are the mainstay therapies for PCOS women (Sathyapalan et al., 2010, Kazerooni et al., 2010; Sangraula et al., 2009). However several findings have shown moderate to severe side effects after prolonged use of some of these pharmacological agents (Corrao et al., 
2004; Hoffmann et al., 2003). Thus, current research is an attempt to focus on alternative therapies to prevent these adverse effects.

Galactomannan is a soluble fibre, indigestible polysaccharides (complex carbohydrates), composed of galactose and mannose. It is obtained from plant Trigonella foenum graceum Linn belonging to family Leguminosae. The soluble dietary fibre fraction, galactomannan is shown to be responsible for hypoglycemic effect of fenugreek seeds and improves the insulin sensitivity (Kamble et al., 2013). It is also reported as antiandrogenic (Aswar et al., 2008), antihyperlipidemic (Boban et al., 2006), antioxidant (Wang et al., 2010). Low molecular weight galactomannans which was used in the present study have already been evaluated for acute oral toxicity, subchronic toxicity and mutagenic potential (Deshpande et al., 2016). Therefore, present study was carried out with an objective to evaluate the effect of galactomannan on letrozole-induced PCOS in adult female Wistar rats.

\section{Materials and Methods}

\section{Experimental Animals}

Female Wistar rats with body weight ranging from 250-300 g and 8 weeks old were procured from the Institute's animal house and were maintained at a temperature of $25 \pm 1^{\circ} \mathrm{C}$ and relative humidity of 45 to 55\% under 12-h light: 12-h dark cycle with free access to food pellets (Pranav Agro Industries Ltd., Sangli, India) and water ad libitum. All experiments were carried out between 09:00 and 17:00 h. The protocol was approved by the Institutional Animal Ethics Committee (IAEC), Sinhgad Institute of Pharmacy, Narhe, Pune, Maharashtra, India (IAEC approval no. SIOP/IAEC/2016/03) constituted according to guidelines of Committee for the Purpose of Control and Supervision on Experiment on Animals. (1139/PO/a/07/CPCSEA). These animals were randomly divided into six groups $(\mathrm{n}=6)$ as normal group, PCOS control group [LTZ $1 \mathrm{mg} / \mathrm{kg}$, per oral], Standard group [CC, $1 \mathrm{mg} / \mathrm{kg}$, per oral], test groups [GM 10, 20 and $40 \mathrm{mg} / \mathrm{kg}$, per oral).

\section{Drugs and Chemicals}

Letrozole (Natco Pharma, Hyderabad), Clomiphene citrate (Maneesh Pharmaceuticals Ltd. Mumbai), Galactomannan was obtained as a gift sample from Indus biotech, Pune and carboxymethyl cellulose was obtained from Burgoyne Burbidges, Mumbai. Other chemicals used in this study were of analytical grade. Glucose, Cholesterol, Triglycerides, HDL and Total protein kits were obtained from Coral clinical systems, India.

\section{Induction of PCOS and Sample Collection}

All the animals except in normal group were orally administered with letrozole $(1 \mathrm{mg} / \mathrm{kg}$, p.o.) dissolved in 0.5 Carboxy Methyl Cellulose (CMC) once daily for 21 days (Kafali et al., 2004). Normal group received vehicle only $(0.5 \% \mathrm{CMC})$. Vaginal smears were collected on daily basis and microscopic evaluation was carried out using Giemsa stain, that confirmed induction of PCOS. After 21 days treatment with letrozole, rats in all groups were administered with respective treatment from day 22 to day 36 (15 days). After 21 days, PCOS control group and after 36 days, animals from other groups were kept on overnight fasting, followed by anaesthesia with diethyl ether. Blood was collected by retro orbital puncture, serum was separated and used for estimation of hormones, glucose and lipid parameters. The animals were then sacrificed and their ovaries and uterus were excised, fat was cleaned off and weighed. After excision, ovaries were made bloodfree, cleaned with ice cold saline and were used for further histopathological studies.

\section{Measurement of Non-Invasive Blood Pressure}

Animals from all the groups were subjected for noninvasive blood pressure. The rat was placed in rat holder in such a way that the tail remains outside. In the pressure cuff, the tail of animal was inserted and pressure cuff was connected to Power lab system (AD instruments, Australia) and blood pressure was measured using lab chart. After assigning the channels, animal was allowed to stabilize for $15 \mathrm{~min}$. The cuff pressure released slowly to measure the BP and ECG.

\section{Estimation of Biochemical Parameters in Blood/Serum}

Blood was centrifuged at $3000 \mathrm{rpm}$ for $30 \mathrm{~m}$; serum was separated and used for the estimation of blood glucose, total cholesterol, LDL, HDL, Triglycerides, hormones like testosterone, LH, FSH, AMH using diagnostic kits. Lipid profiles were estimated using enzymatic kits obtained from Coral clinical systems, India. Friedewald's equation was used to calculate LDL-cholesterol (LDL-C). Serum FSH was assayed by sandwich Radioimmune Assay (RIA), serum LH and testosterone were measured by competition RIA. All the serum samples for hormonal parameter estimation were outsourced to Vedha pathology lab, Pune.

\section{Statistical Analysis}

Results are expressed as the mean \pm Standard Error of the Mean (SEM). Statistical analysis was carried out by one way followed by Tukey's multiple range tests; and two-way Analysis of Variance (ANOVA) followed by Bonferroni post hoc test by using the software Graph 
Pad Prism trial version 5.0 (Graph Pad Software, Inc., La Jolla, CA, USA). A value of $\mathrm{p}<0.05$ was considered to be statistically significant.

\section{Results}

\section{Effect of GM on Body Weight and Blood Sugar Level (BSL)}

No significant changes were seen in the body weight of rats measured on every week. Significant reduction in BSL was observed with GM $(10 \mathrm{mg} / \mathrm{kg})$ and CC (1 mg/kg) compared to LTZ control (Fig. 1).

\section{Effect of GM on Lipid Profile}

Significant increase in serum triglycerides, cholesterol and LDL and decreased HDL concentration was found in the animals of control group treated with letrozole as compared to the animals in normal group. Treatment either with standard or test drugs significantly reduced the concentration of serum triglycerides, cholesterol and LDL concentration and increased HDL when compared with animals in letrozole treated control group (Fig. 2).

\section{Effect of GM on Serum Hormonal Profile}

Significant reduction in FSH and AMH was observed in the animals of letrozole treated control group as compared to the animals in normal group. Whereas, the concentration of FSH and AMH in animals in standard and test groups were found to be increased when compared with the animals in letrozole treated control group. From the Fig. 3, it is observed that there is a significant increase in the level of testosterone and luteinizing hormone in the letrozole induced control group. Treatment either with standard, clomiphene citrate or galactomannan reverted back these hormone concentrations as compared to the animals in control group (Fig. 3).

\section{Effect of GM on Ovary Weights}

Weight of ovaries in the animals of letrozole treated control group was found to be significantly increased when compared with normal animals. Weight of ovaries in animals treated either with standard or test drug was significantly reduced as compared to LTZ control (Fig. 4 and 5).

\section{Effect of GM on Blood Pressure and Heart Rate}

No significant changes were observed in blood pressure and heart rate was observed in any treatment group, hence data not shown.

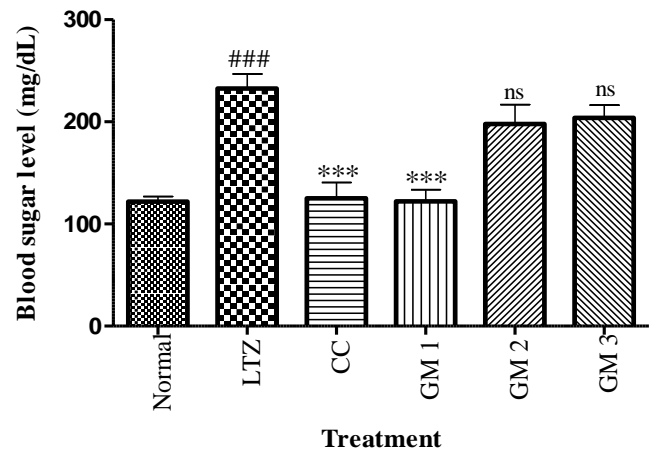

Fig. 1: Effect of GM on blood sugar level. Data was expressed as mean \pm SEM $(n=6)$. Statistical significance was determined using one way ANOVA followed by Tukey test. \#\#\# p<0.001 compared to normal, $* * * \mathrm{p}<0.001$, ns: Non-significant compared to LTZ control

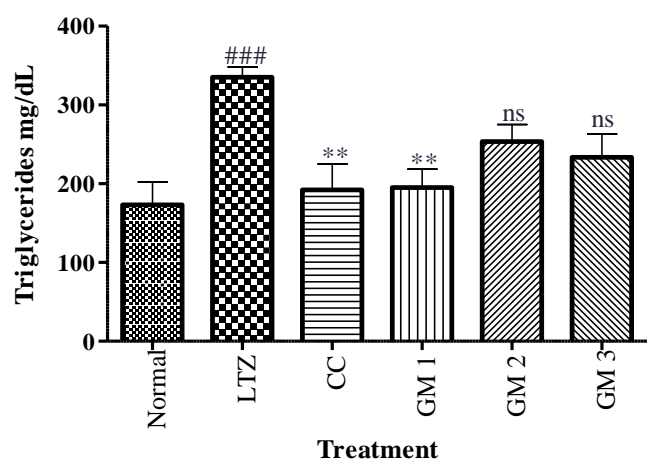

(A)

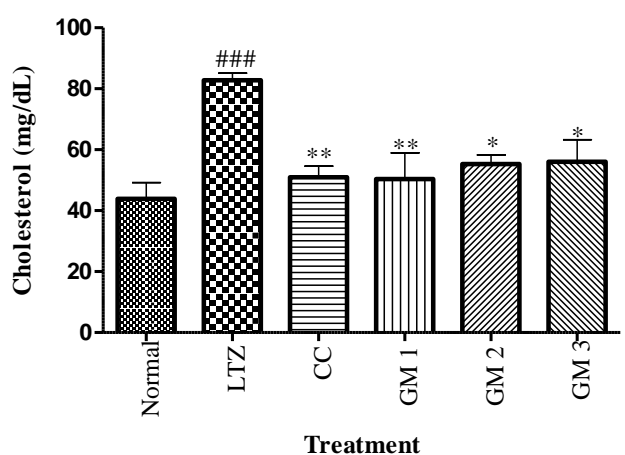

(B)

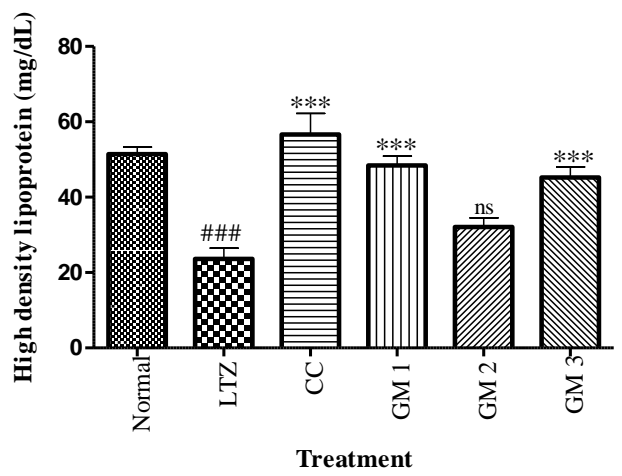

(C) 


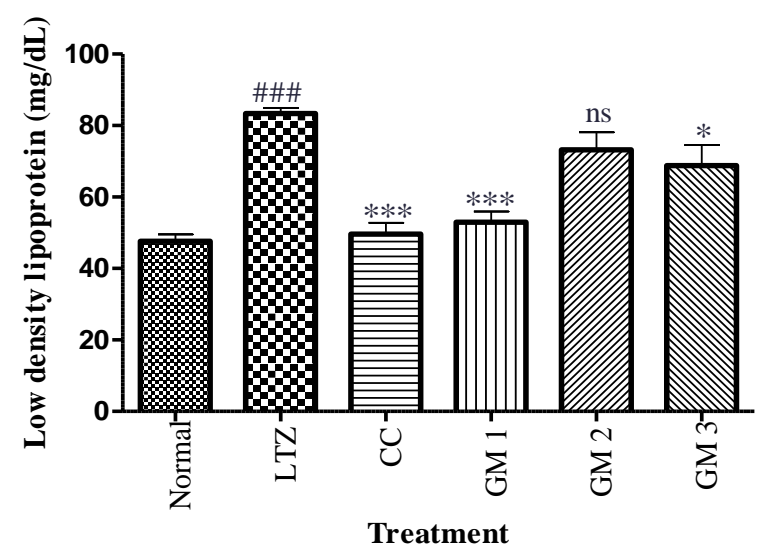

(D)

Fig. 2: Effect of GM on lipid profile. A. Triglycerides; B. Serum cholesterol; C. High Density Lipoprotein; D. Low Density Lipoprotein. Data was expressed as mean \pm SEM $(n=6)$. Statistical significance was determined using one way ANOVA followed by Tukey test. \#\# $\mathrm{p}<0.001$ compared to normal, $\quad * * * \mathrm{p}<0.001, \quad * * \mathrm{p}<0.01, \quad * \mathrm{p}<0.05$ and $\mathrm{ns}$ : Non-significant compared to LTZ control

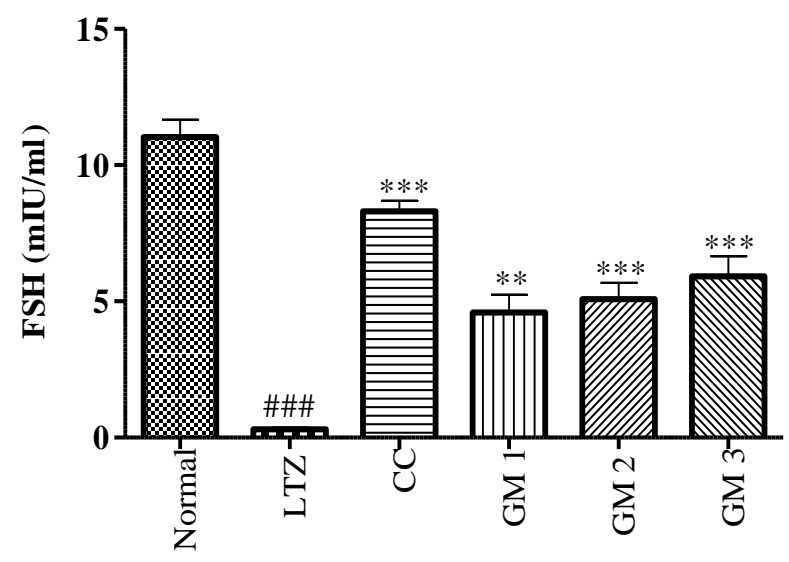

Treatment

(A)

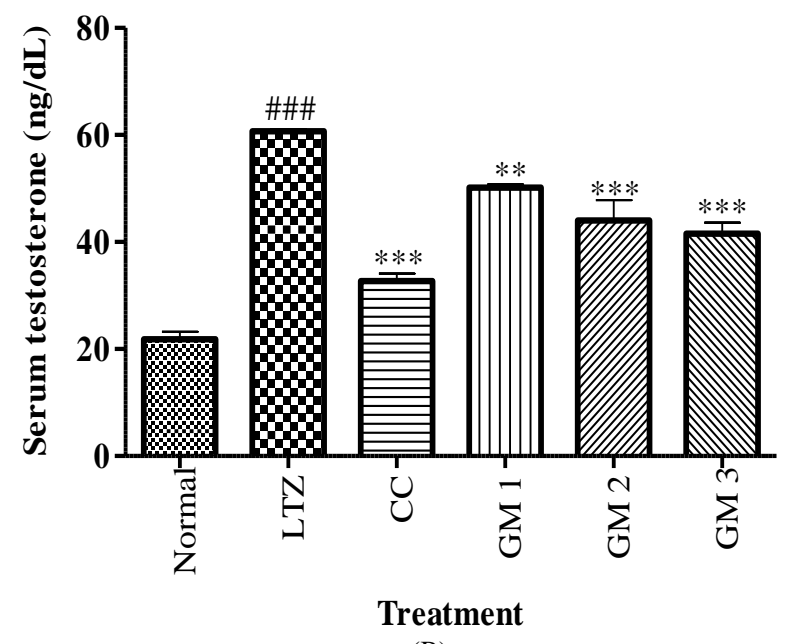

(B)

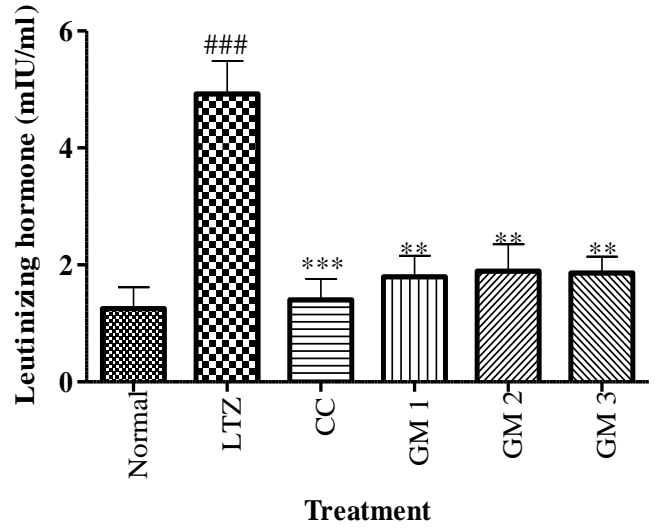

(C)

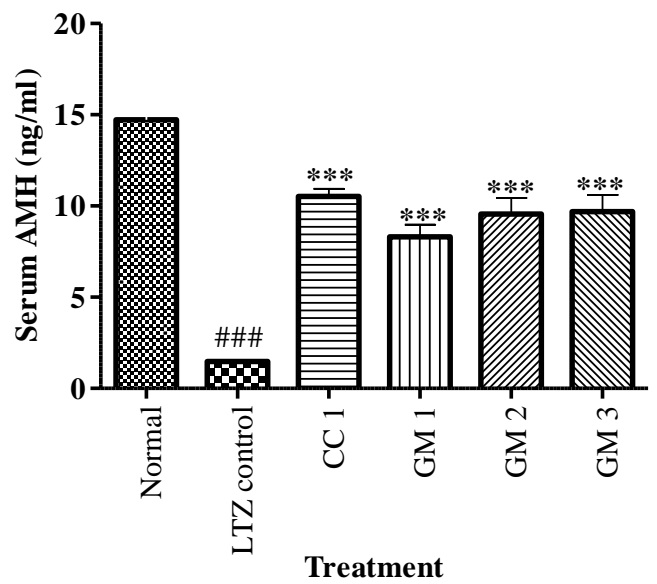

(D)

Fig. 3: Effect of GM on serum hormonal profile, A. Follicle Stimulating Hormone (FSH); B. Testosterone; C. Luteinizing Hormone (LH); D. Anti-Mullerian Hormone $(\mathrm{AMH})$. Data was expressed as mean \pm SEM $(n=6)$. Statistical significance was determined using one way ANOVA followed by Tukey test. \#\#\# p<0.001 compared to normal, $* * * \mathrm{p}<0.001, * * \mathrm{p}<0.01, * \mathrm{p}<0.05$ and $\mathrm{ns}$ : Non-significant compared to LTZ control

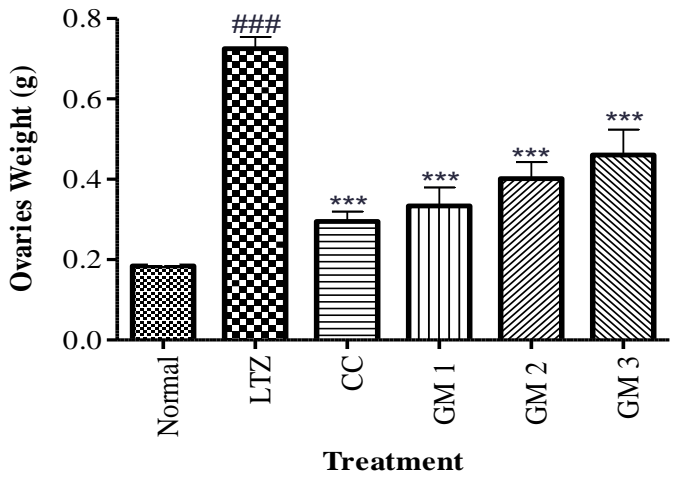

Fig. 4: Effect of GM on ovaries weight. Data was expressed as mean $\pm \operatorname{SEM}(n=3)$. Statistical significance was determined using one way ANOVA followed by Tukey test. \#\#\# $\mathrm{p}<0.001$ as compared to normal and $* * * \mathrm{p}<0.001$ compared to LTZ control 


\section{Effect of GM on Histopathology of Ovaries}
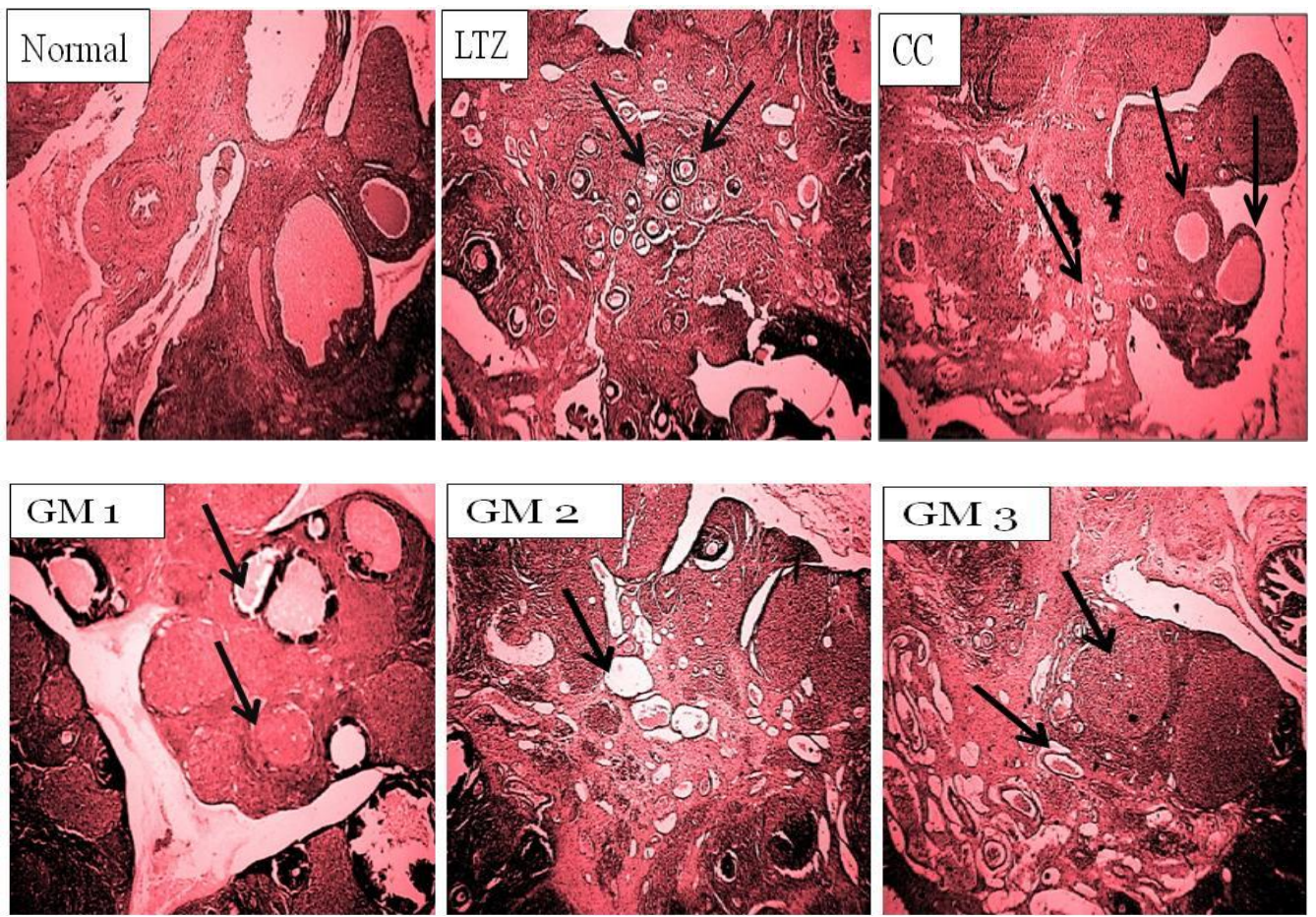

Fig. 5: Histopathology images of ovaries obtained from Normal rats showing no cysts growth, LTZ control rats showing number of cysts on the ovary, CC (1 mg/kg) and Galactomannan $(10,20,40 \mathrm{mg} / \mathrm{kg})$ showed less number of cysts as compared to LTZ control group

\section{Discussion}

PCOS is a common heterogeneous endocrinological and metabolic disorder with multiglandular pathophysiology in reproductive women, leading to infertility or reduced fertility. Clinical manifestations of PCOS include infrequent/absent menstruation, abdominal obesity and signs of hyperandrogenism, that include acne and insulin resistance. Long term consequences include increased risk of endometrial cancer, type- 2 diabetes mellitus, dyslipidemia, hypertension and cardiovascular disorders (Teede et al., 2010). Chemicals such as androgens (Dehydroepiandrosterone (DHEA), testosterone propionate and 5-dihydrotestosterone, estradiol valerate, antiprogesterone and letrozole are used to induce PCOS in mice and rats. Among them, letrozole-induced PCOS rats were the most suitable model for experiment. Letrozole is a nonsteroidal aromatase inhibitor that suppresses the estrogen biosynthesis in PCOS-like symptoms in rats, resulting in androgen accumulation (Daneasa et al., 2016). As a result, ovarian cells cannot produce estrogen and an increased circulating androgens leads to polycystic ovary with an abnormal follicular cycle (Kafali et al., 2004; Caldwell et al., 2014, Walters et al., 2012). Follicular atresia and abnormal follicular development is observed due to induced elevation of androgen levels inside the ovary (Choi et al., 2005). Anovulation causes disturbance in the feedback signalling from ovarian sex hormones to the hypothalamus and pituitary which in turn, affect the release of the Gonadotropin-Releasing Hormone (GnRH) disturbing normal release of LH and FSH (Teede et al., 2010). Feng et al. (2013) reported that the increased level of LH may also be due to impaired feedback of estrogen and in turn proliferates theca cells, inducing PCOS condition. In the present study, we observed non-significant weight gain, increased blood sugar level, acyclicity, hyperandrogenism, increased LH and cystic ovarian morphology in letrozole-induced rats. Treatment either with clomiphene citrate or galactomannan significantly decreased the level of $\mathrm{LH}$ and testosterone in the treatment group of rats, thus protecting the ovaries from excess proliferation of the theca cells and helps in the prevention of PCOS. Testosterone is derived partly from adrenal glands and partly from ovaries in normal women. However, in case of women with PCOS, whole source of testosterone is ovary as stated by Marshall (2001). This increased androgen further leads to hyperinsulinemia and further insulin resistance by altering the glucose metabolism. In our present investigation, elevated levels of testosterone might have caused significant increase in the insulin resistance and an increased in the blood glucose level 
as seen in the letrozole treated animals. Oral administration of galactomannan at all doses significantly decreased level of testosterone indicating suppressive action on insulin resistance and further decrease in blood glucose level which are in line of previous findings (Kamble et al., 2013; Evans et al., 1992; Hamden et al., 2010).

Galactomannans are polysaccharides consisting of a mannose chain substituted with galactose side chains. Galactomannan has potential to alter the glycemic and lipid levels in rats (Evans et al., 1992). It also has effect on digestive enzymes related to diabetes, hyperlipidemia and liver dysfunction. Galactomannan exhibited promising therapeutic effects on postprandial hyperglycemia and hyperlipidemia and significantly prevented diabetic toxicity in the liver and kidney (Hamden et al., 2010). Fenugreek galactomannan, when dissolved in water in stomach, swells instantly and increases viscosity. As a result, glucose absorption is delayed, which leads to decreased blood sugar level. In the present study, an attempt has been made to unravel the adjuvant benefit of galactomannan in letrozole induced PCOS.

In the present study, letrozole induced rats showed increase in body weight and after treatment with clomiphene citrate and galactomannan; it showed reduction in body weight. PCOS women are more susceptible for type 2 diabetes mellitus because of the insulin resistance, so glycemic index was performed on day 21 and day 36. In the present study, Letrozole induced rats showed increased blood sugar level and treatment groups showed decrease in blood sugar level on the above-mentioned days. PCOS is a metabolic disorder related to hyperlipidemia, lowest dose of galactomannan $(10 \mathrm{mg} / \mathrm{kg}$ ) showed decrease in triglycerides, total cholesterol, LDL and increased HDL. Apart from lipid profile, variation in total protein was also estimated on the last day of study, treatment either with standard or test drugs significantly reduced total protein concentration.

Anti-Mullerian Hormone (AMH) is a member of the transforming growth factor- $\beta$ family. It serves as a marker for PCOS as it expressed in all steps of folliculogenesis. The AMH level is 2-3 times higher in case of PCOS and displays good sensitivity and specificity for the diagnosis of PCOS. Decrease in AMH suggests the decrease number of follicles which are responsible for dysmennorrhoea, PCOS and infertility. While increased testosterone level in female body shows male characteristic in female like unwanted facial and body hair growth. In this study, letrozole treated rats showed decrease in FSH and $\mathrm{AMH}$ while galactomannan and clomiphene citrate showed increase in FSH and AMH. Letrozole treated rats showed significant increase in $\mathrm{LH}$ and testosterone while treatment with galactomannan and clomiphene citrate showed decrease in LH and FSH levels. Increased blood sugar level and lipid profile is generally associated with variation in cardiovascular parameter. In the present study, no significant variations on cardiovascular parameters (blood pressure and heart rate) were recorded.

The ovaries of rats were further subjected to histopathology. Letrozole treated rats showed increased number of cysts on ovaries when compared with normal. Clomiphene citrate and galactomannan treatment showed decrease in number of cysts. Galactomannan at the dose of $10 \mathrm{mg} / \mathrm{kg}$ showed significant decrease in number of cysts. The probable mechanism of galactomannan to decrease cysts and its beneficial effects on PCOS may be because of its role in increasing the sensitivity of glucose for cellular intake and possibly by increasing insulin concentration. Also the finding supports to decrease testosterone and LH which may contribute to increase ovulation.

\section{Conclusion}

The results presented in our findings suggest that galactomannan has many useful effects on hormonal changes in rats with PCOS. These changes significantly aid the recovery of the oestrus cycle, the number of developed follicles and ovarian morphology in rats with PCOS. These findings may be ascribed to multiple pharmacological activities of galactomannan like estrogenic, antihyperlipidemic, antioxidant and hypoglycemic effects which could be useful in managing PCOS condition and prevent ovarian cell dysfunction, ovulation and thereby improving fertility.

\section{Acknowledgement}

Authors are thankful to Hon. Prof. M. N. Navale Sir and Dr. K. G. Bothara Sir for providing the platform for the conduct of experiments.

\section{Author's Contributions}

Ms. Utkarsha Deshpande: Execution of Experimental Work.

Dr. Urmila Aswar: Writing of manuscript

Dr. Manoj Aswar: Plan of work and writing of manuscript

\section{Ethics}

All the animals used in the experiments were sanctioned from Institutional Animal Ethics Committee (IAEC) as per the rules and regulations of CPCSEA, New Delhi.

\section{References}

Aswar, U., Mohan, V., Bhaskaran, S. \& Bodhankar, S. (2008). Study of galactomannan on androgenic and anabolic activity in male rats. Pharmacology online, 56-65. 
Boban, P. T., Nambisan, B. \& Sudhakaran, P. R. (2006). Hypolipidaemic effect of chemically different mucilages in rats: A comparative study. British Journal of Nutrition, 96, 1021-1029. doi.org/10.1017/BJN20061944

Caldwell, A., Middleton, L., Jimenez, M., Desai, R., Mcmahon, A., Allan, C., Handelsman, D. \& Walters, K. (2014). Characterization of reproductive, metabolic and endocrine features of polycystic ovary syndrome in female hyper androgenic mouse models. Endocrinology, 155, 3146-3159. doi.org/10.1210/en.2014-1196

Choi, S. H., (2005). Psychological side-effects of clomiphene citrate and human menopausal gonadotrophin. Journal of Psychosomatic Obstetrics \& Gynecology, 26, 93-100. doi.org/10.1080/01443610400022983

Corrao, G., Zambon, A., Bertù, L., Botteri, E., Leoni, O. \& Contiero, P. (2004). Lipid lowering drugs prescription and the risk of peripheral neuropathy: An exploratory case-control study using automated databases. Journal of Epidemiology \& Community Health, 58, 1047-1051. doi.org/10.1136/jech.2003.013409

Daneasa, A., Cucolas, C., Lenghel, L. M., Olteanu, D., Orasan, R. \& Filip, G. A. (2016). Letrozole vs estradiol valerate induced PCOS in rats: Glycemic, oxidative and inflammatory status assessment. Reproduction, 151, 401-409. doi.org/10.1530/REP-15-0352

Deshpande, P., Mohan, V., \& Thakurdesai, P. (2016). Preclinical safety evaluation of low molecular weight galactomannans based standardized fenugreek seeds extract. EXCLI journal, 15, 446.

https:/www.ncbi.nlm.nih.gov/pmc/articles/PMC508 3966/

Evans, A., Hood, R., Oakenfull, D. \& Sidhu, G. (1992). Relationship between structure and function of dietary fibre: A comparative study of the effects of three galactomannans on cholesterol metabolism in the rat. British Journal of Nutrition, 68, 217-229. doi.org/10.1079/BJN19920079

Expert Panel on Detection, E. (2001). Executive summary of the third report of the National Cholesterol Education Program (NCEP) expert panel on detection, evaluation and treatment of high blood cholesterol in adults (adult treatment panel III). Jama, 285(19), 2486-2497. doi.org/10.1001/jama.285.19.2486

Feng, Y., Li, X., \& Shao, R. (2013). Genetic modeling of ovarian phenotypes in mice for the study of human polycystic ovary syndrome. American journal of translational research, 5(1), 15. https://www.ncbi.nlm.nih.gov/pmc/articles/PMC356 0480/
Hamden, K., Jaouadi, B., Carreau, S., Bejar, S. \& Elfeki, A. (2010). Inhibitory effect of fenugreek galactomannan on digestive enzymes related to diabetes, hyperlipidemia and liver-kidney dysfunctions. Biotechnology and Bioprocess Engineering, 15, 407-413. doi.org/10.1007/s12257-009-3037-9

Hoffmann, I. S., Roa, M., Torrico, F. \& Cubeddu, L. X. (2003). Ondansetron and metformin-induced gastrointestinal side effects. American Journal of Therapeutics, 10, 447-451. doi.org/10.1097/00045391-200311000-00012

Kafali, H., Iriadam, M., Ozardalı, I. \& Demir, N. (2004). Letrozole-induced polycystic ovaries in the rat: A new model for cystic ovarian disease. Archives of Medical Research, 35, 103-108. doi.org/10.1016/j.arcmed.2003.10.005

Kamble, H., Kandhare, A. D., Bodhankar, S., Mohan, V. \& Thakurdesai, P. (2013). Effect of low molecular weight galactomannans from fenugreek seeds on animal models of diabetes mellitus. Biomedicine \& Aging Pathology, 3, 145-151. doi.org/10.1016/j.biomag.2013.06.002

Kazerooni, T., Shojaei-Baghini, A., Dehbashi, S., Asadi, N., Ghaffarpasand, F. \& Kazerooni, Y. (2010). Effects of metformin plus simvastatin on polycystic ovary syndrome: A prospective, randomized, double-blind, placebo-controlled study. Fertility and sterility, 94, 2208-2213. doi.org/10.1016/j.fertnstert.2009.11.045

Legro, R. S., Gnatuk, C. L., Kunselman, A. R. \& Dunaif, A. (2005). Changes in glucose tolerance over time in women with polycystic ovary syndrome: A controlled study. The Journal of Clinical Endocrinology \& Metabolism, 90, 3236-3242. doi.org/10.1210/jc.2004-1843

Maqbool, M., Dar, M. A., Gani, I. \& Geer, M. I. (2019). Insulin Resistance and Polycystic ovary Syndrome: A Review. Journal of Drug Delivery and Therapeutics, 9, 433-436. doi.org/10.22270/jddt.v9i1-s.2275

Marshall, K. (2001). Polycystic ovary syndrome: Clinical considerations. Alternative Medicine Review, 6, 272-272.

Nair, M., Pappachan, P., Balakrishnan, S., Leena, M., George, B. \& Russell, P. S. (2012). Menstrual irregularity and poly cystic ovarian syndrome among adolescent girls-a 2 year follow-up study. Indian Journal of Pediatrics, 79, 69-73. doi.org/10.1007/s12098-011-0432-y

Norman, R. J., Dewailly, D., Legro, R. S. \& Hickey, T. E. (2007). Polycystic ovary syndrome. The Lancet, 370, 685-697. doi.org/10.1016/S0140-6736(07)61345-2

Sangraula, H., Paudel, K. \& Sharma, M. (2009). Metformin and troglitazone in the treatment of female infertility associated with polycystic ovarian syndrome. Journal of the Nepal Medical Association, 48, 335-339. doi.org/10.31729/jnma.350 
Sathyapalan, T., Kilpatrick, E. S., Coady, A. M. \& Atkin, S. L. (2010). Atorvastatin pretreatment augments the effect of metformin in patients with Polycystic Ovary Syndrome (PCOS). Clinical endocrinology, 72, 566-568. doi.org/10.1111/j.1365-2265.2009.03678.x

Teede, H., Deeks, A. \& Moran, L. (2010). Polycystic ovary syndrome: A complex condition with psychological, reproductive and metabolic manifestations that impacts on health across the lifespan. BMC medicine, 8, 41.

doi.org/10.1186/1741-7015-8-41
Walters, K. A., Allan, C. M. \& Handelsman, D. J. (2012). Rodent models for human polycystic ovary syndrome. Biology of reproduction, 86, 149, 1-12. doi.org/10.1095/biolreprod.111.097808

Wang, X., Wang, J., Zhang, J., Zhao, B., Yao, J. \& Wang, Y. (2010). Structure-antioxidant relationships of sulfated galactomannan from guar gum. International Journal of Biological Macromolecules, 46, 59-66. doi.org/10.1016/j.ijbiomac.2009.10.004

Wild, R. A. (2002). Long-term health consequences of PCOS. Human reproduction update, 8, 231-241. doi.org/10.1093/humupd/8.3.231 